Originalveröffentlichung in: Honold, Alexander; Simon, Ralf (Hrsgg.): Das erzählende und das erzählte Bild, München 2010, S. 55-83 (Eikones)

\title{
Erscheinung statt Erzählung
}

Werner Busch

Das klassisch-idealistische Kunstkonzept, das, ob wir es wollen oder nicht, unseren Begriff von Kunst bis heute prägt, wurde zuerst in der italienischen Kunsttheorie des 16. Jahrhunderts formuliert, um dann an der französischen Akademie des 17. Jahrhunderts seine kanonische Form zu finden, vor allem in Gestalt einer verbindlichen Gattungshierarchie. Es ist, verkürzt gesagt, das Ergebnis einer Mischung aus platonischem bzw. neoplatonischem Idee-Begriff und aristotelischem Nachahmungsbegriff, seine begriffliche Ausgestaltung vollzog es nach rhetorischem Vorbild, sowohl, was die Definition der Kompositionsprinzipien angeht, als auch das Konzept des Dekorums. Zugleich aber verpflichtete es sich dem Horazischen Diktum des ut pictura poesis. Das Bild verstanden als Einlösung eines vorgängigen Textes, eines concetto, mit ihm eigenen Mitteln, die allerdings einen schrittweisen Nachvollzug, man könnte sagen, einen textförmigen Nachvollzug, befördern sollen "Lisez la peinture", forderte Poussin und entwickelte Strategien, uns schrittweise zur Sinnerschließung durchs Bild zu führen. Der Bildsinn hat sich erfüllt, wenn wir einer Bilderzählung haben folgen können und durch ihre ideale Verbildlichung zu einer Nutzanwendung etwa in religiöser, politischer oder moralischer Hinsicht 
geführt werden, anders ausgedrückt: wenn es uns möglich wird, die bloße Erzählung zu transzendieren. ${ }^{1}$

Daneben hat es von Anfang an eine zweite Auffassung von Kunst gegeben mit gleicher Geltungsdauer, die uns nur deswegen nicht als ein Konzept im Bewusstsein ist, weil sie nicht eine eigene, in Traktatform geronnene Theorie hervorgebracht hat. Von daher sehen wir sie, noch einmal, ob wir wollen oder nicht, bis heute aus dem Blickwinkel klassischer Theorie und tradieren das Verdikt, das aus diesem Blickwinkel einem Gegenentwurf in allem und jedem gegenüber notwendig formuliert werden musste. Aus klassischer Sicht ist dieses Gegenmodell antiklassisch, antiidealistisch, antiakademisch, und es reicht von daher ein einziges Totschlagargument, um es zu diskreditieren: Seine Kunst, wenn man überhaupt davon sprechen kann, ist bloße Naturnachahmung, Handwerk ohne höheren Anspruch. Es gibt die Natur, wie sie ist, nicht, wie sie sein soll. Die klassische Kunst basiert auf dem Disegno, der Zeichnung, dem immateriellsten Medium überhaupt, und ist daher der Idee am nächsten. Wenn die manieristische Kunsttheorie zwischen Disegno interno und Disegno esterno scheidet, und damit geistige Vorstellung und zeichnerische Umsetzung gleichermaßen mit dem Disegno-Begriff belegt, dann kann der deutsche Begriff "Entwurf « diese beiden Pole in sich fassen, zudem aber das entäußerte Produkt als etwas definitiv Abgeschlossenes, Endgültiges bezeichnen. Als in Linien Festgelegtes hat es sich vom Künstler gelöst und scheinbar Objektivität erlangt. Seine Umsetzung ins Gemälde, seine farbige Fassung, ist eine bloße Materialisierung des zuvor schon vollgültig Vorhandenen, da es die Idee in sich trägt. ${ }^{2}$

Diesen Prozess der Materialisierung der Idee hat die akademische Kunstauffassung in Werkstufen ausdifferenziert. Über eine erste zeichnerische, flüchtige Ideenskizze führt der Weg zur Konkretion über einen ersten sorgfältigen Gesamtentwurf, die Modellstudien zur Klärung des Einzelnen und einen endgültigen Entwurf oder Karton an der Schwelle zur Übertragung ins Gemälde, dem noch eine Farbstudie vorangehen kann. Die Figuration jedenfalls ist gefunden und ändert sich bei der Übertragung ins Gemälde im Prinzip nicht mehr, sie bleibt als der eigentliche geistige Kern erhalten. Da sie diesen Kern in sich birgt, kann das Resultat über sich hinausweisen.

Es fragt sich nun, wie dem gegenüber das zweite Modell verfährt. Was soll man unter bloßer Naturnachahmung in einem medialen Prozess verstehen, wo doch, was festzustellen eine 
Binsenweisheit darstellt, jede künstlerische Aneignung eine Transformation darstellt? Das Verblüffende nun ist, dass die Vertreter der unklassischen Position den Vorwurf der bloßen Naturnachahmung weitgehend akzeptiert, ihn allerdings in eine besondere Tugend umgemünzt und sich dabei ganz bewusst antiker Rechtfertigungsstrategien bedient haben. Offenbar gab es keine andere Sprachregelung. Die Praxis der unklassischen Künstler seit Tizians unterscheidet sich radikal von derjenigen der klassischen Künstler. Wenn Raphael, als der Urvater der klassischen Künstler, in seinem kurzen Leben, das gerade einmal 37 Jahre dauerte, Hunderte von Zeichnungen gemacht hat, die sich problemlos den aufgeführten Zeichenkategorien der Entwurfspraxis fügen, dann kennen wir von Tizian, der deutlich über 80 Jahre alt wurde, knapp 50 Zeichnungen, und sie fügen sich den Kategorien weitgehend nicht. Selbst die Zeichnungen, die die Forschung als Entwurfszeichnungen deklariert, scheinen nicht vor, sondern während des malerischen Prozesses entstanden zu sein, an Punkten, an denen der Künstler mit einer gemalten Lösung nicht zufrieden war und Alternativen zeichnerisch ausprobieren wollte. Das heißt, in dieser Tradition findet die Bildfindung im malerischen Prozess selbst statt. Und noch nicht einmal dies ist ganz richtig, denn zu einer eigentlichen Findung, als eines definitiven Resultates, einer Vollendung, kommt es nicht wirklich.

Das liegt an der Art der Gestaltung. Die unklassischen Künstler übersetzen nicht etwas in der Form bereits Vorherbestimmtes, sondern generieren die Form und damit den Sinn im malerischen Prozess. Eben deswegen finden sich bei ihnen zahlreiche Pentimenti, korrigierende Übermalungen, Veränderungen, Verschiebungen, erneute Antworten auf andernorts auf dem Bildkörper vorgenommene Ergänzungen etc. Von daher haben auch ihre Bilder keinen geschlossenen, abgeschlossenen Farbkörper, ist die Farbmaterie unterschiedlich weit entwickelt und zwar so weit, wie für die jeweilige Aussageabsicht nötig.

Um ein Extrembeispiel zu nennen: In Tizians spätem Marsyas [Abb. 1] hat man an manchen Partien bis zu dreißig Übermalungen festgestellt; an anderen bleibt die Untermalung stehen. $\mathrm{Zu}$ leicht hat man sich hier wie andernorts in Tizians Spätwerk damit beholfen, das ganze späte CEuvre für unvollendet zu erklären. Doch wie lassen sich dann vorhandene Signaturen oder die Tatsache, dass Tizians späte Pietà schon einmal an Ort und Stelle, an ihrem Bestimmungsort, dem projektierten Grabmal Tizians gewesen 


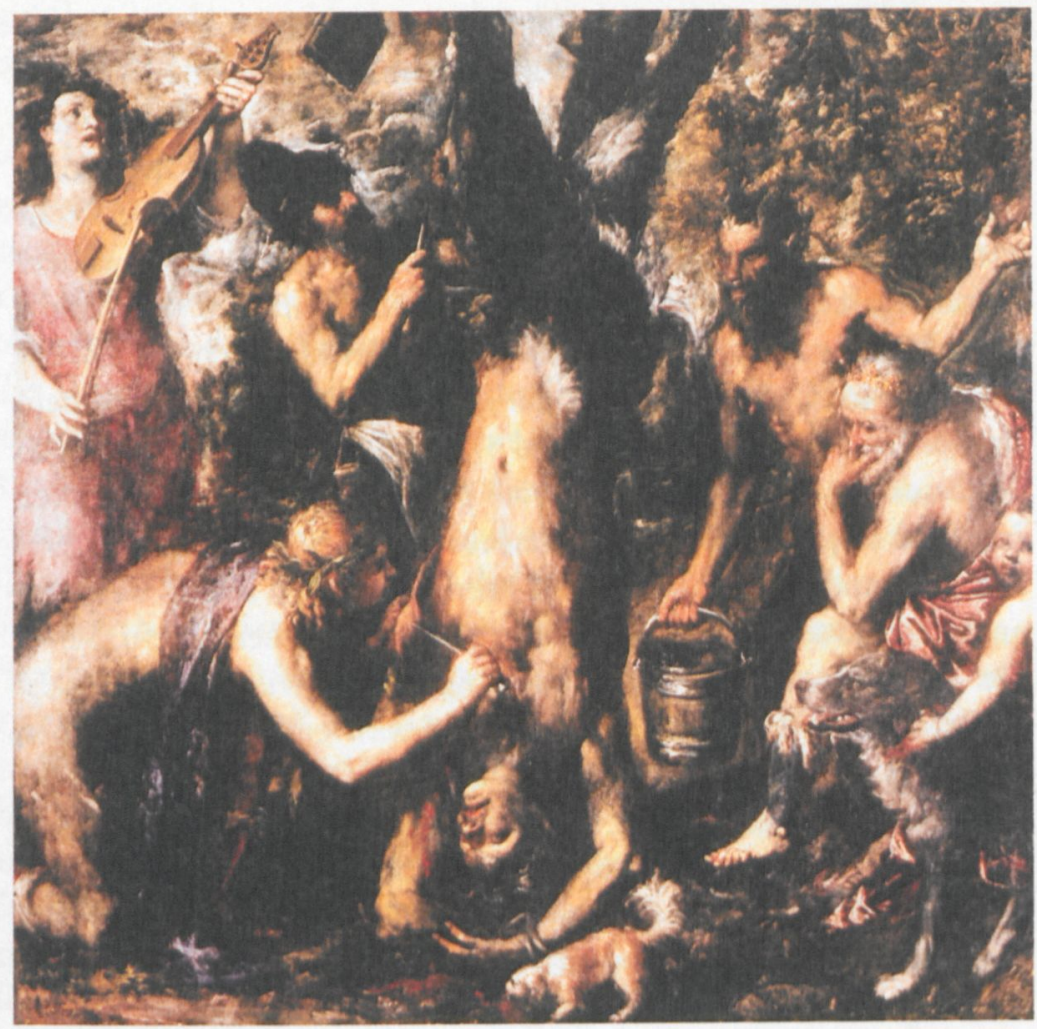

1 Tizian, Die Schindung des Marsyas, um 1570, Ö। a. L., $220 \times 204 \mathrm{~cm}$, Erzbistum Olomouc, Erzbischöflicher Palast Kroměřiž, Gemäldegalerie ist, erklären, bevor sie von den Kirchenoberen für unzureichend erklärt und wieder entfernt wurde? Beim Marsyas sind die Dinge noch komplexer. Wir können noch nicht einmal mit Sicherheit sagen, wer eigentlich Apoll ist-der kniend Schindende vorne? - oder ob er gar zweimal vorkommt? Denn wer ist der »lira da braccio «Spieler ganz links, er scheint am häufigsten übermalt worden zu sein; offenbar konnte Tizian hier keine abschließende Klarheit gewinnen, und so ließ er ihn stehen, er deutet Apollinisches an, selbst wenn er in dieser Gestalt nicht in den unmittelbaren, sondern nur den mittelbaren Zusammenhang der Schilderung der Schindung gehört. Und auch der Midas, der noch dazu Tizians Porträtzüge zu tragen scheint, ist nicht leicht in den Kontext der Erzählung zu integrieren. Er ist nicht der inkompetente Richter im Musikwettstreit, dem zur Strafe Eselsohren wachsen, sondern ein melancholisch über den Gang der Dinge Nachsinnender. So wird von links wie von rechts die Geschichte als Geschichte aufgehoben, ergibt sich keine unmittelbare Textlogik, vielmehr ist die Erzählung in einem auf Dauer gestellten Moment der Reflexion, des 
Nachdenkens über unausweichliches Schicksal aufgehoben. ${ }^{3}$ Man kann auch sagen, sie zerfällt in Teile, die nicht konsequent auf ein Zentrum bezogen sind, vielmehr Aspekte der Sache entfalten.

Was können wir vorläufig aus dem Geschilderten schließen? Jede Ưbermalung, ja, jeder zusätzliche Pinselstrich im Werk stiftet neuen Sinn, verändert das bisher Ausgedrückte. Kommt diese Tatsache zu Bewusstsein, so wird das Werk tendenziell unabschließbar. Gelegentlich ist die Farbmaterie bei Tizian kaum gestaltet und bleibt in ihrem Materiecharakter erfahrbar; auf diese Weise werden uns die entstehende Form und der sich daraus ergebende Sinn vorgeführt; nicht mehr, aber auch nicht weniger. Denn das in der Bosse Stehengelassene, das nicht weiter Gestaltete, das Nicht-Abgeschlossene ist notwendig zu den Betrachtenden hin offen, fordert ihren Anteil ein, wobei es ihnen möglich ist, bei der Fortschreibung des Erfahrenen diesen oder jenen Weg einzuschlagen. Zudem entwerten sowohl Unabgeschlossenheit als auch nicht auf einen Punkt gebrachte Korrekturen die Ikonographie. Ihre letztliche Uneindeutigkeit, oder anders ausgedrückt: die 


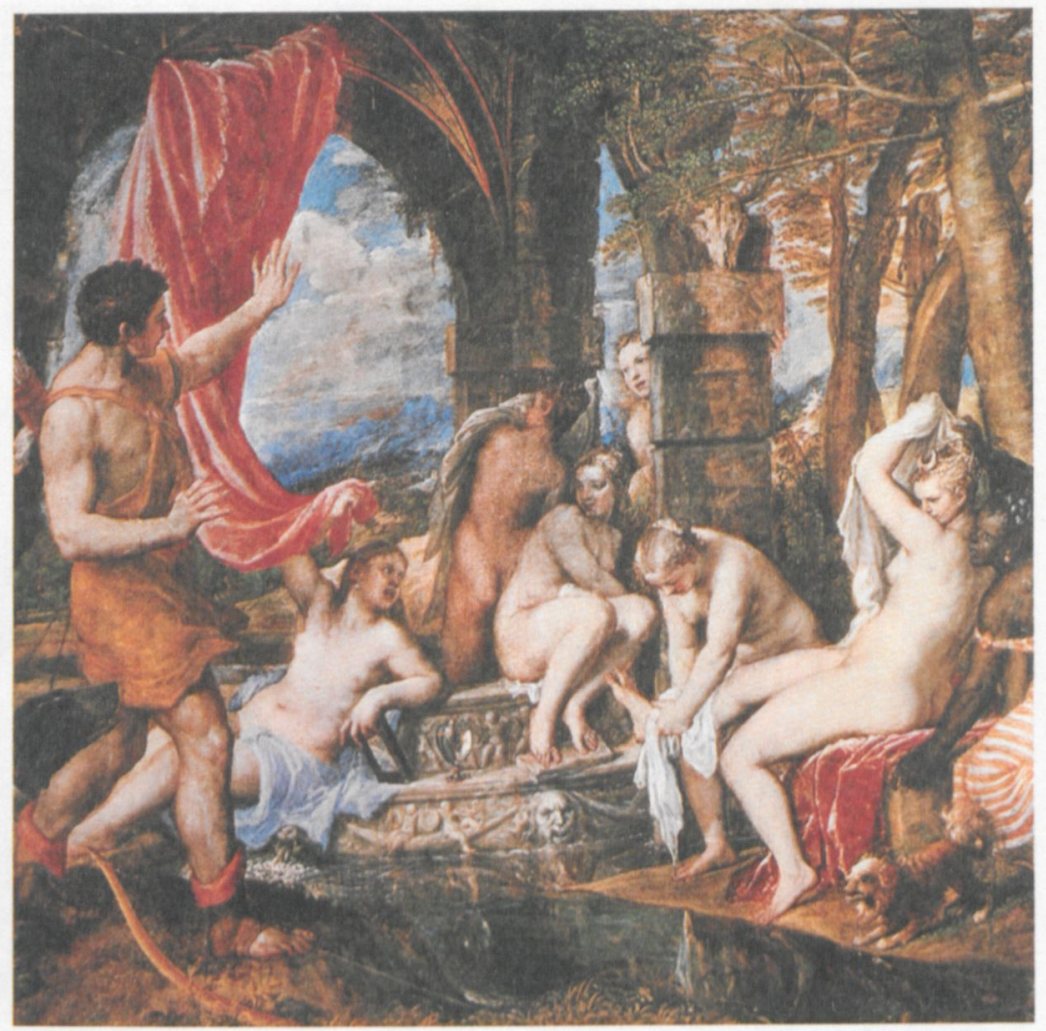

2 Tizian, Diana und Aktäon, 1556-59, Öl a.L., $190,3 \times 207 \mathrm{~cm}$, Edinburgh, National Gallery of Scotland
Eröffnung verschiedener, nicht notwendig harmonierender, zu einer Synthese führender Bedeutungsstränge, entfaltet verschiedene Facetten ein- und derselben Sache und zwar in der Weise, dass eine kontinuierliche Erzählung davor verstummt.

Doch noch etwas geschieht auf diese Weise und erweist sich als durchaus paradox. Zum einen, da keine eigentliche Handlungsentwicklung veranschaulicht wird, erscheint das Bild stillgestellt für eine Reflexion darüber, was veranschaulicht ist. Zum anderen: Da die Dinge und der Sinn als Entstehende bzw. als Werdender vorgeführt sind, der Farbkörper Höhen und Tiefen, offene und geschlossene Partien hat, ist das Bild für die Wahrnehmung in Bewegung, es atmet, ist lebendig, und diese Lebendigkeit übertragen wir auch auf das Gezeigte, das ebenfalls zu leben und zu weben scheint. Der Farbkörper der Spätwerke Tizians ist ein Produkt extrem reduzierter Farbigkeit. $\mathrm{Zu}$ finden sind allein noch tonale Abstufungen weniger Grundtöne. Ein Gelb-Braun-Kontinuum mit dem Mittelton Ocker, das ins Grüne oder aber ins Rote hin gebrochen sein kann, bestimmt den Bildkörper. Das heißt, auch die 


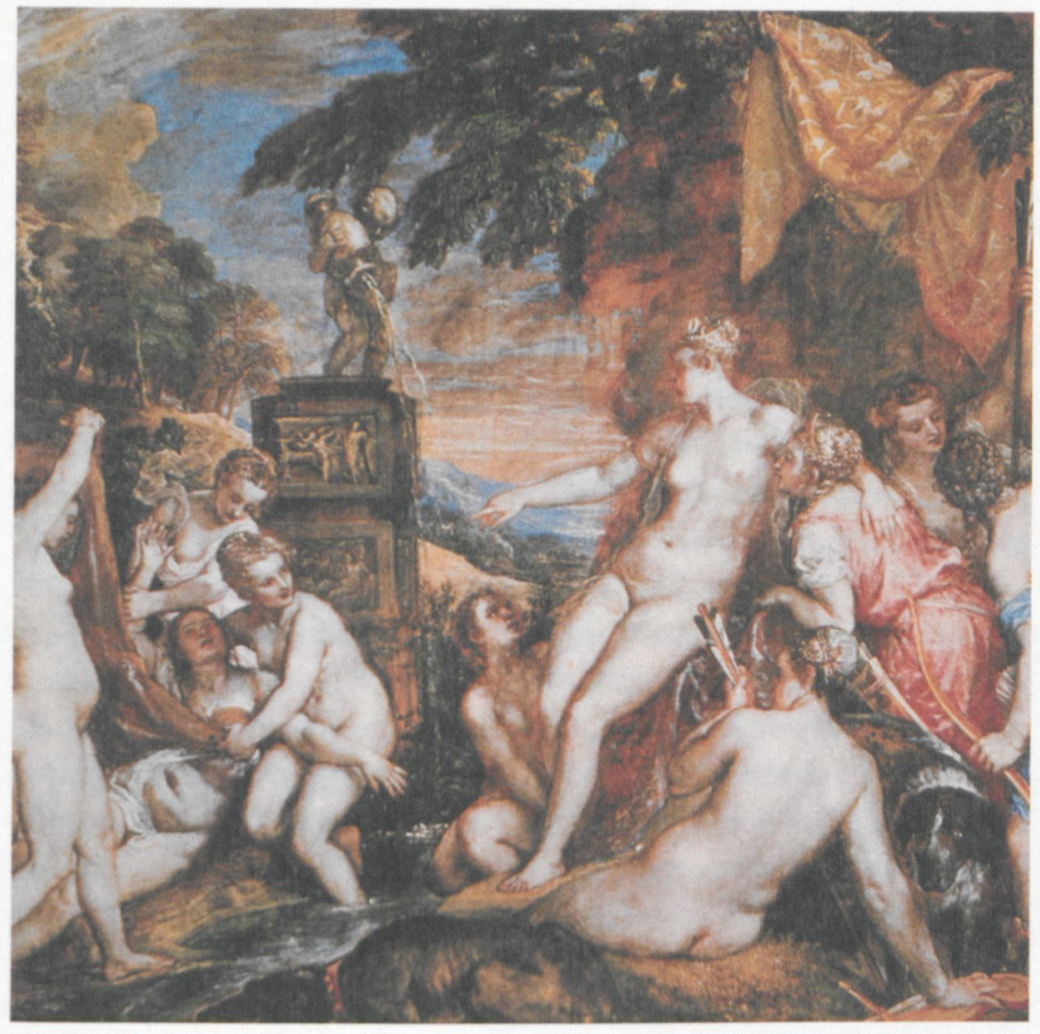

Farbe ist als bloß schwach Auftauchende markiert, auch sie müssen wir dem Gemälde erst einschreiben, auch die Farbe wird als Entstehende vorgeführt. ${ }^{4}$

Nun gibt es im Spätwerk Tizians zwei Bilder, die eine verblüffende Ausnahme darstellen: Sie weisen eine ausgesprochene Buntfarbigkeit auf, die sonst eigentlich nur im Frühwerk Tizians zu finden ist. Es sind die beiden Dianenbilder [Abb. 2 u. 3], die zur sogenannten Poesia für Philipp II. gehören, entstanden zwischen 1556 und $1559 .{ }^{5}$ Sie wurden gemeinsam an Philipp geschickt, sind in vielfacher Hinsicht Pendants. Sie haben das gleiche Rahmenthema, das darin besteht, dass jeweils verbotenes Tun von Diana bestraft wird, obwohl in beiden Fällen bei den Bestraften, Aktäon bzw. Callisto, keine individuelle Schuld vorliegt, wie schon die Quelle, Ovids Metamorphosen, betont. Die Exegese konnte dies anders sehen: unkeusches Sehen und unkeusches Handeln verdienen strengste Strafe. ${ }^{6}$ Bei Ovid geht es um unvermeidliches Schicksal und seine einzig denkbare Form der Aufhebung durch Metamorphose. Die Betroffenen werden in Blumen verwandelt oder zu den Sternen 
entrückt. In vielem folgt Tizian dem Ovidschen Text, kennt zudem genau das ikonographische Vokabular der Szenen aus den Ovid-Illustrationen, wobei für ihn die Übersetzung des Ovidschen Textes ins Volgare durch seinen Freund Lodovico Dolce am wichtigsten ist; sie ist zuerst 1553 erschienen, 1558 in einer fünften Auflage, versehen mit den Holzschnittillustrationen von Giovanni Rusconi, die, im Gegensatz zur älteren Tradition, weitgehend auf Simultandarstellungen verzichten. ${ }^{7}$ So scheint hier eine klassische Historie vorzuliegen mit durchaus entwickelter Erzählhaltung, zumal sich eine wahre Fülle von durchaus über Ovid hinausgehenden Textverweisen nachweisen lässt, die die Forschung weitgehend übersehen hat.

Und doch ist Vorsicht geboten, denn der Witz, wenn man so sagen darf, bei Tizians Bildern besteht darin, dass die Textreferenzen zwar erkennbar werden, es sich jedoch beinahe durchgehend um subtile Abweichungen von der jeweiligen Textvorlage handelt, in denen Tizian seine besondere Auffassung hervorkehrt - und zwar in zweierlei Hinsicht: bezogen auf die Farbe und bezogen auf das Verhältnis von Natur und Kunst. Die Buntfarbigkeit hat eine direkte Quelle und ihr folgt Tizian durchaus: in Albertis Della Pittura bzw. in De Pictura von 1435/36 heißt es im Kapitel darüber, wie die Maler die Farben richtig aufzutragen haben: »Ich möchte, dass auf einem Gemälde alle Gattungen [von Farben] und jede ihrer Arten zu sehen sind, damit sie die Betrachtung anreizen und vergnügen. Anmut wird sich dort einstellen, wo nebeneinanderliegende Farben voneinander sehr verschieden sind; also wenn du Diana malen würdest, wie sie ihr Gefolge anführt, so sei das Gewand der einen Nymphe grün, das einer anderen weiß, das einer weiteren rosarot, das einer anderen wiederum safrangelb, und so sei eine jede von unterschiedlicher Farbe; derart, dass die hellen Farben immer neben verschiedenen anderen dunklen Farben zu liegen kommen. Durch diesen Kontrast wird die Schönheit der Farben strahlender und anmutiger sein. Es gibt nämlich eine bestimmte Freundschaft unter den Farben, sodass die Verbindung der einen mit der anderen ihnen beiden Würde und Anmut verleiht. Rosarote Farbe zwischen Grün und Himmelblau gibt allen Ehre und Ansehen. Weiß bereitet nicht nur neben Aschgrau und Safrangelb, sondern fast neben allen [Farben] Freude. Dunkle Farben besitzen unter hellen eine nicht geringe Würde, und ebenso umgeben helle [Farben] vorteilhaft dunkle. Der Maler wird seine Farben so anordnen, wie ich gesagt habe. $\aleph^{8}$ 
Man kann vor allem Tizians Diana und Aktäon durchaus als Einlösung der Albertischen Farbempfehlungen sehen: das rosarote Tuch vor Aktäon ist in der Tat von Grün und Himmelblau gerahmt und das weiße Tuch der Diana, mit dem sie sich zu bedecken sucht, trägt in sich in den verschatteten Zonen bereits Grau, verstärkt zum Aschgrau in der schwarzen Dienerin hinter ihr, die zudem ein weiß und safrangelb, also krokosfarben gestreiftes $\mathrm{Ge}$ wand trägt. Und dennoch, was Alberti meint und was etwa noch in einem klassischen Bild wie Domenichinos Dianenjagd direkt befolgt wird ${ }^{9}$, ist Primärfarbigkeit, klare Farbe neben klarer Farbe. Tizian dagegen bricht die Farben vielfältig im Licht. Das Licht und das Dunkel können Farben aufzehren, im Licht bis zu Weiß entfärben, im Dunkel bis zu Schwarz trüben. Licht liegt auf den Tüchern, Dunkel breitet sich auf dem Wasser vor dem Brunnen aus. In Glas und Spiegel können die Farben vielfach gesammelt und gebrochen sein. So weist uns die Buntfarbigkeit auf die literarische Quelle, scheint ihr ostentativ zu folgen, um dann zu demonstrieren, dass dies nicht mit Notwendigkeit in zeichenhafte Primärfarbigkeit zu münden hat, dass vielmehr Farbharmonie im tonalen Kosmos nur entsteht, wenn die Farben gebrochen werden, um ein atmosphärisches Kontinuum zu stiften. Mag hier die Abweichung von der Quelle noch indirekter Natur sein, die Referenz auf zwei andere Quellen arbeitet bewusst und direkt mit ihrer Sinnverkehrung.

So sehr Tizian im Detail der Hauptquelle, Ovids Metamorphosen, folgt, in einem bezeichnenden Punkt weicht er auch von ihr ab. In der Übersetzung Breitenbachs heißt es zum Hain der Diana:

»Hier lag eine umschattete Höhle im hintersten Winkel, keinerlei Schöpfung der Kunst; die Natur, in eigener Erfindung, hatte ein Kunstwerk geformt: aus lebendigem Bimsstein und leichtem Tuff

einen Bogen gezogen, der hier an der Stelle gewachsen. Lieblich rauschte zur Rechten ein Quell; es floss in ein weites Becken, von grasigem Rand umgürtet, das leuchtende Wasser. « ${ }^{10}$

Indem Tizian den Ovidschen Text in sein Gegenteil verkehrt-nicht wie im Text beschrieben, bringt die Natur eine kunstähnliche Architektur hervor, vielmehr ist seine Grotte ein Werk von Menschenhand, das aber doch, wie sein Verfallscharakter zeigt, wieder in Natur übergeht-indem er dies tut, hinterlässt er eine 
deutliche Signatur seiner Auffassung vom Verhältnis von Kunst und Natur, in diesem besonderen Falle durch die Architekturtheorie Serlios im vierten Buch gerechtfertigt, der schon die gotischen Gewölbeformen, die Tizian hier überraschenderweise verwendet, vor allem aber die Rustika, die kaum behauenen Quader, für kunstlos erklärt, der Natur am nächsten: halb ist sie, die Rustika, ein Werk der Natur, halb der Kunst ("parte opera di natura, e parte opera di artefice «). ${ }^{11}$ Zugleich greift Tizian ein Motiv auf, das insbesondere Aretino unter ausdrücklichem Bezug auf Tizian in allen Varianten verwendet, auch eben in der Umkehr: nicht nur lässt er Tizians Kunst wie Natur erscheinen, seine Figuren scheinen sich zu bewegen, zu atmen, zu sprechen, das gemalte Fleisch scheint zu zittern etc., zumal sich Entsprechendes in Dolces L'Aretino zu Tizian findet. ${ }^{12}$

In seinem berühmten Brief an Tizian von 1544 spricht Aretino vielmehr von einem Natureindruck und lässt die Häuser Venedigs wie gemalt erscheinen (»di materia arteficiata «), wo sie doch aus Stein seien. Aretinos Absicht ist eindeutig, denn im Folgenden betont er, allein Tizians Malerei sei in der Lage, das Atmosphärische mitzumalen, die tote Natur für die Erfahrung zu verlebendigen. So erschöpft sich Tizians Malerei nicht in der bloßen Naturnachahmung, sondern wie der göttliche Schöpfer vermag er es, den Dingen Pneuma, Lebensatem, einzuhauchen. Die Natur wird zur Malerei, Tizian aufgrund seiner schöpferischen Potenz zu Natur, oder, wie es an anderer Stelle bei Aretino präzisierend heißt, Tizian schaffe die Idee einer anderen Natur. ${ }^{13}$

Am wichtigsten in unserem Zusammenhang ist jedoch der rote Vorhang, der wie ein Aufmerkzeichen für Gefahr einsteht: Schreitet Aktäon dennoch voran, erblickt er Diana, so ist es um ihn geschehen. Das mag seine Bildfunktion gewesen sein, seine späte korrigierende Einführung ins Gemälde dürfte das ganze Bild umgekrempelt haben: Aktäon rückt weiter vor, die Nymphe, die nun den Vorhang hält, hatte zuvor Aktäon angeschaut, jetzt wendet sie sich zurück und blickt auf Diana, aber auch Diana ist weiter gedreht worden, und schließlich ist die Dienerin erst in späterem Zustand zu einer Schwarzen geworden. Wohl auch jetzt erst wurde, um das bukranionartige Hirschgeweih aufnehmen zu können, aus der ursprünglichen Säule, hinter der sich die neugierig auf Aktäon schauende Nymphe verbirgt, ein Pfeiler. Das Hirschgeweih verweist bereits auf Aktäons Schicksal. So kommt dem Vorhang wahrlich umwälzende Funktion zu. Die Herkunft dieses Motivs 
aus literarischem Kontext hat man übersehen, zudem seine paradoxe Umkehr durch Tizian.

Es stammt aus der Ars poetica des Horaz und wiederum stand Tizian eine naheliegende Übersetzung zur Verfügung, erneut durch seinen Freund Lodovico Dolce, aber diesmal bereits von 1536, bezeichnenderweise dem gemeinsamen Freund Aretino gewidmet. ${ }^{14}$ Am berühmten Anfang des Briefes über die Dichtkunst, von dem immer nur die Bemerkungen zu den in der Regel unstatthaften Chimären der Maler zitiert werden, heißt es, um die Forderung des Dekorums zu illustrieren: "Oft wird an gewichtige Anfänge und große Versprechen hier und da ein Lappen von Purpur, dass weithin er leuchte, angeflickt, wenn man den Hain und Altar der Diana beschreibt und wie sich ein munteres Bächlein durch liebliche Ackerflur schlängelt, oder den Rheinstrom oder den Regenbogen abbildet. Doch war dafür just nicht der richtige Platz (»sed nunc non erat hic locus«).«

Und wenig später heißt es: "Kurz, es sei das Werk, wie es wolle, nur soll es geschlossen und einheitlich sein «. ${ }^{15}$ Eben dies wird es im Spätwerk von Tizian immer weniger. Den weithin leuchtenden roten Lappen jedoch, den Horaz als entrée in ein Werk wie den Hain der Diana für abwegig und unpassend hält, bringt Tizian ostentativ an, denn seine künstlerische Behandlung kann ihn rechtfertigen. Erneut führt Tizian uns vor, was seine Kunst vermag. Möglich ist der rote Vorhang nur jenseits von Albertis Farbordnung, weil die malerische Faktur, die Tizian stehen lässt, und die Farbbrechung ihn trotz seiner Buntfarbigkeit einbinden und er zugleich eine überzeugende Bildfunktion gewinnt.

Nun hat Tizian Philipp II. in einem Brief von 1559 davon berichtet, dass er an einem dritten Dianenbild [Abb. 4] arbeite. ${ }^{16}$ Es ist nie nach Spanien gelangt, womöglich, weil es sich radikal von den beiden anderen unterscheidet. Denn seine Farbigkeit ist vollkommen zurückgenommen, es ist fast monochrom und zudem scheint sein Motiv keine direkte textliche Grundlage zu haben. Die Forschung hat es ob seiner Monochromie und seiner passagenweise nicht weit entwickelten peinture wieder für unvollendet erklärt mit dem durchaus richtigen Hinweis darauf, dass Tizian nicht selten Bilder in angefangenem Zustand an die Wand gestellt und sie erst viel später bei Bedarf, etwa durch einen Auftrag, weiter gemalt habe. ${ }^{17}$ Und dennoch: Zum einen greifen bei einem Künstler wie Tizian die Kategorien "vollendet « und »unvollendet « nicht mehr; jede Fortsetzung der Arbeit am Bild verändert dieses inhaltlich. 


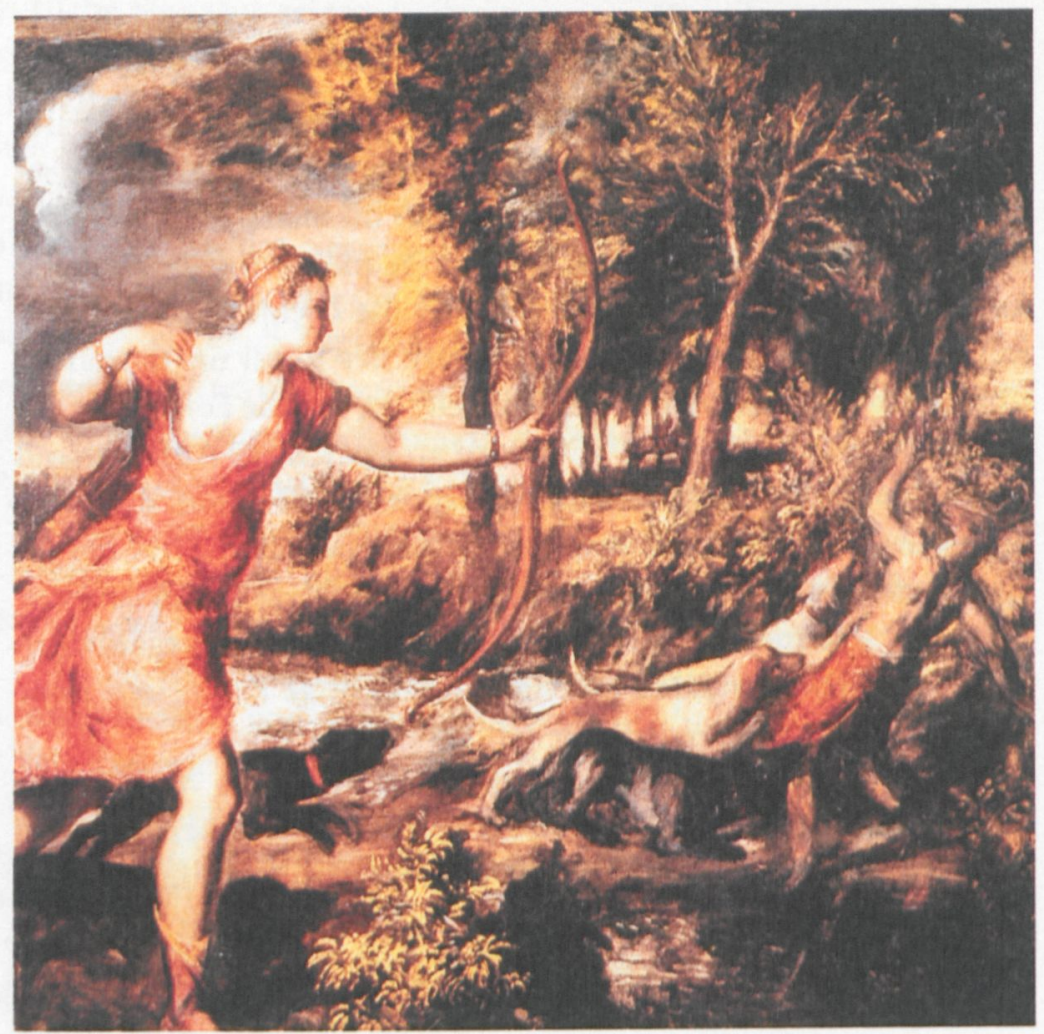

4 Tizian, Der Tod des Aktäon, um 1559-1568, Öl a.L., $179 \times 189 \mathrm{~cm}$, London, National Gallery
Rein theoretisch kann eine hinreichende Absicht in einem relativ frühen Entwicklungsstadium bereits erreicht werden. Insofern haben Tizians Bilder Palimpsestcharakter: Jede neue Überarbeitung hebt die alte auf, macht sie aber nicht ungeschehen. Zum anderen hat die farbige Reduktion eine doppelte Funktion.

Auf der einen Seite erweist sich Tizian als neuer Apelles. Schon Karl V. hatte ihn 1533 so bezeichnet, und Tizian selbst spielt im Briefwechsel mit Philipp II. darauf an. ${ }^{18}$ Von Apelles heißt es bei Plinius, er habe ein dunkles Pigment benutzt, um die Farben zu dämpfen, das berühmte atramentum, über dessen Status allerdings nicht wirklich Klarheit herrscht. Wichtiger noch: Apelles habe die Farben auf drei oder vier reduziert, Rot, Gelb, Schwarz und Weiß, er habe nicht die vielen neu erfundenen Pigmente benutzt, um sein Werk modisch aufzuschicken, er könne vielmehr mit Wenigem Alles darstellen. ${ }^{19}$ Schließlich habe Apelles die zu große Vollendung der Werke des Protogenes kritisiert. ${ }^{20}$ Man konnte darin eine Rechtfertigung der Tizianschen colpi sehen, der in der Faktur stehen gelassenen Pinselhiebe. ${ }^{21}$ Dass diese Rechtfertigung 
aus dem antiken Schrifttum zu beziehen war, ließ sie geeignet erscheinen, als Gegengewicht gegen die klassische Ideenvorstellung als Voraussetzung für Kunstproduktion zu dienen.

Auf der anderen Seite ist der Charakter des Unvollendeten von Tizians Bildern, oder besser: des Vorläufigen, nicht nur ein Hinweis auf den Produktionsprozess, sondern er hält das Bild in einer Schwebe, zwischen Anfang und Ende, pathetisch gesprochen: zwischen Leben und Tod. Dieses Vorläufige, das im Modus des Chiaroscuro daherkommt, allein Licht und Schatten in tonaler Abstufung braucht, weckt in allen Teilen seiner Erscheinung, wie weit oder wenig entwickelt auch immer, das Bedürfnis nach Fortschreibung. Sinnbeimessung, aber auch bloße Entzifferung, und das Resultat dieses Rezeptionsprozesses sind nicht in eine textliche Sinneinheit zu übersetzen, sie sind fortwirkend anregend, eröffnen die verschiedensten Wege, mögliche Parallelwege. Das Bild hat nicht allegorischen Charakter, wird nicht zum Symbol, sondern ist eine Metapher, die Spielraum lässt. Zugleich aber ist es, was es ist: unterschiedlich weit gestaltete Materie, und führt damit den 


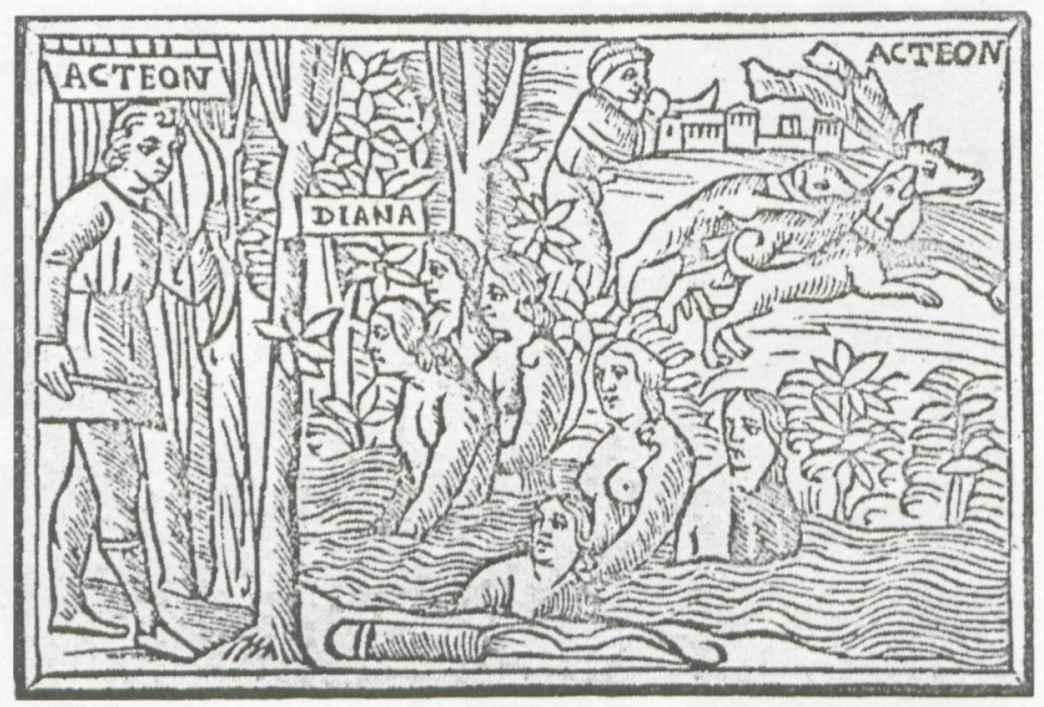

5 III. zu Ovid, Metamorphosen, Venedig 1513, fol. XIXro, Holzschnitt, Diana und Aktäon
Prozess von Gestaltung überhaupt vor, macht zusehends für die mediale Seite der Kunst bewusst.

Doch das dritte Dianenbild ist noch durch etwas anderes ausgezeichnet: Es hat keine Textgrundlage. Die Forschung hat es mit Irritation zur Kenntnis genommen. Die dargestellte Szene kommt weder bei Ovid, noch in den Illustrationen vor. Ich vermute, Tizian kombiniert einen textlichen Hinweis, eine Bildquelle mit einem anderen Dianenthema, und eine Illustration und macht etwas gänzlich Neues daraus. Als Diana den Eindringling Aktäon wahrnimmt, bedauert sie, so heißt es bei Ovid, dass sie Pfeil und Bogen abgelegt und nicht zur Hand hat ${ }^{22}$; von daher legen die Illustrationen sie gelegentlich ostentativ im Bildvordergrund aus [Abb. 5]. ${ }^{23}$ An späterer Stelle im Text, als die Verwandlung des Aktäon durch das Spritzwasser der Diana bereits eingesetzt hat, flieht er, die Hunde fallen über ihn her und zerreißen ihn, das stellen die Illustrationen gelegentlich dar [Abb. 6] ${ }^{24}$ So scheint Tizians dritte Szene so etwas wie Dianas Imagination darzustellen, sie wünscht sich, sie habe Aktäon zu Tode gejagt, mit ihren Pfeilen vernichtet. Der imaginäre Charakter könnte dadurch bei Tizian zum Ausdruck gekommen sein, dass Dianas Bogen die Sehne und gar erst der Pfeil fehlen. Das Verfolgungsmotiv mit der hoch aufgerichteten, unaufhaltsamen Jägerin mag sich von Pietro Peruginos Kampf der Venus mit Diana herleiten, zu verstehen als genereller Kampf von "Castitas « und »Voluptas«. ${ }^{25}$ Eben diese Dimension sah die Exegese der Ovidschen Aktäon-Geschichte in seinem unkeuschen Blick 


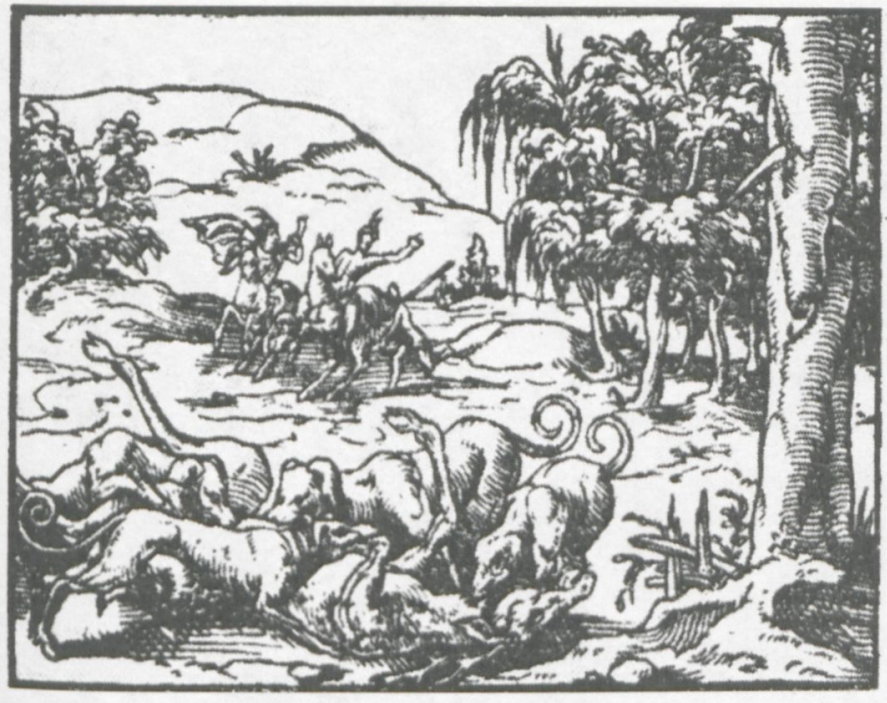

auf die Göttin der Keuschheit verkörpert. ${ }^{26}$ Andererseits ist Tizian ausgesprochen textgetreu, denn die Ovidsche Quelle liefert eine Rechtfertigung für das sonderbare labile Standmotiv des Aktäon, das nicht nur vom Ansturm der Hunde herrührt. Denn auf der Flucht erblickt er sein verwandeltes Gesicht, sieht das Geweih auf seinem Haupt in einer Wasserspiegelung, er stockt, denkt an die Schande zuhaus. Dann heißt es bei Ovid: „Während er schwankt, ersehn ihn die Hunde [... « ${ }^{27}$ Dieses Schwanken scheint Tizian zu verbildlichen, erst dadurch ist Aktäon endgültig verloren. Die Hunde verkörpern offenbar den vernichtenden Pfeil der Diana und nicht nur, wie es Karel von Mander möchte, die Lüste des Aktäon.

Das Tiziansche Medienbewusstsein geht in der Geschichte der Kunst nicht wieder verloren, ja, es kann im 18. Jahrhundert zum eigentlichen Thema des Bildes werden, wie kurz am Beispiel Gainsboroughs verdeutlicht werden soll, dem die Zeitgenossen "an extravagant looseness of pencilling « attestierten. ${ }^{28}$ Thomas Gainsboroughs Diana und Aktäon [Abb. 7], ein Spätwerk von 1785, stellt eine absolute Ausnahme im Euvre dieses Künstlers dar, es ist seine einzige Historie überhaupt. Gainsborough hat sich Zeit seines Lebens zum Antiliteraten stilisiert, behauptet, kaum je ein Buch gelesen zu haben, Figürliches in seinen Landschaftsbildern sei nur dazu da, "to create a little business for the Eye ${ }^{29}{ }^{29}$ Seine Vorliebe galt nicht dem Buch, sondern der Musik, die er selbst auf hohem Niveau betrieb, vor allem aber war er mit zahlreichen Musikern befreundet. Es ist überliefert, dass er abends nach des

6 III. zu Ovid, Metamorphosen, Lyon 1557, No.43, Holzschnitt von Bernard Salomon, Der Tod des Aktäon

7 Nächste Doppelseite: Thomas Gainsborough, Diana und Aktäon, 1784-86, Öl a.L., $158,1 \times 188 \mathrm{~cm}$, London, Her Majesty Queen Elizabeth II.

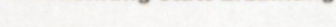




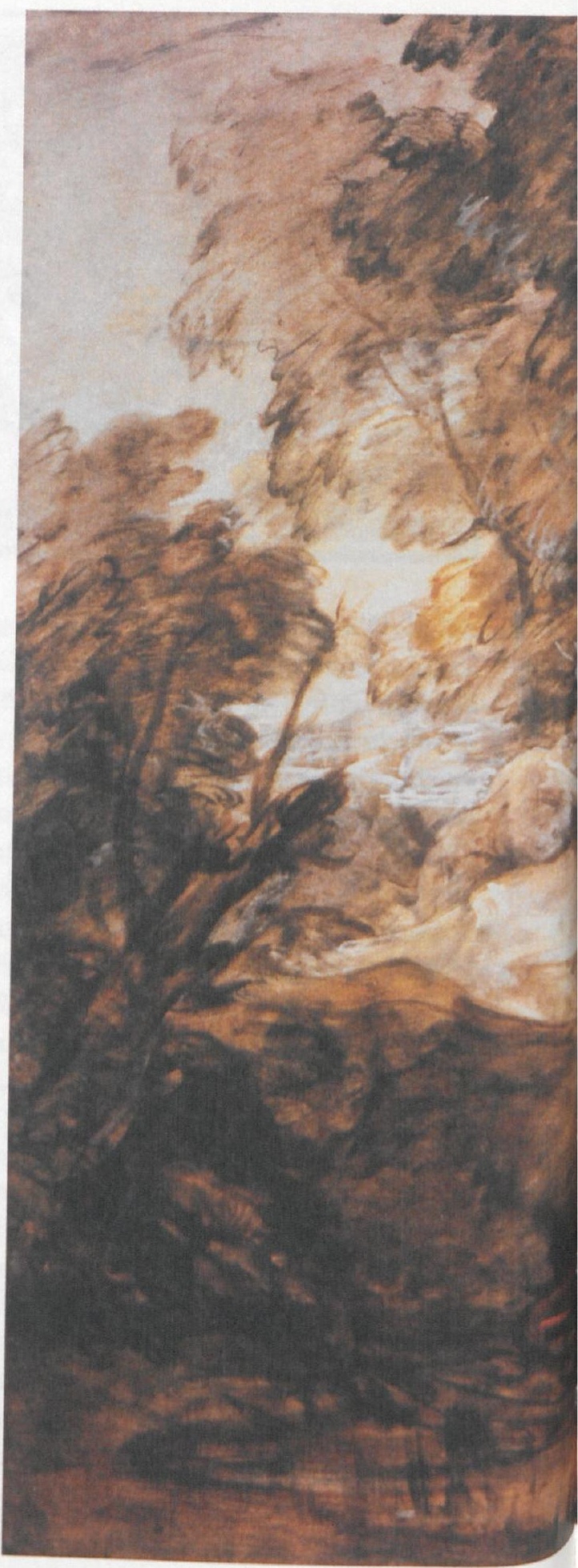



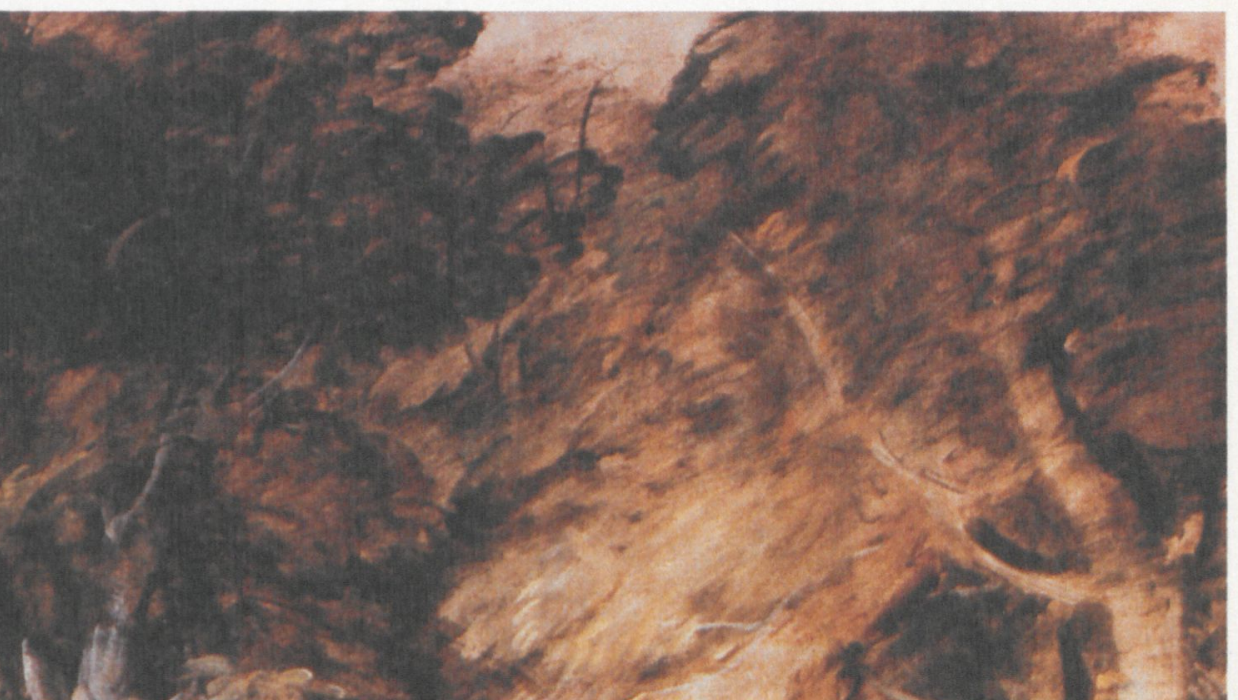

2. Insese

$6+10^{2}=0$ 
Tages Gemäldearbeit, vor allem der von ihm gehassten Porträtmalere ${ }^{30}$, unter Freunden zu Musikbegleitung Landschaften mit dem Kreidestift auf dem Papier entworfen habe, ohne sich recht zu besinnen, ohne direkten Naturbezug. Im Grunde handelt es sich um mehr oder weniger gegenstandslose Strukturierungen des Zeichenbogens, eine flüchtige Massenandeutung. Viele Blätter füllte er auf diese Weise: allein vom "mood» der Musik gestimmt. Die Blätter wurden am nächsten Morgen auf dem Boden ausgebreitet, gelungene, ausgewogene, ausdrucksvolle Massenverteilungen ausgewählt und womöglich als Ausgangspunkt für ein Landschaftsgemälde genutzt; der Rest wurde verworfen. Landschaft und Gestimmtheit sollten so übereinkommen, ausgedrückt in skizzenhafter Malerei, bei der der Gegenstand geradezu irrelevant wurde. ${ }^{31}$

Warum dann eine mythologische Historie? Man hat das Bild, dessen Partien in der Tat unterschiedlich weit entwickelt sind und das nie ausgestellt wurde, unvollendet genannt. Manches ist in der bloßen Untermalung stehen geblieben, ein Teil des Figürlichen ist so wenig konkretisiert, dass er kaum zu identifizieren ist. Und doch ist dieses Gemälde vorbereitet wie kein anderes. Allein drei Gouachen existieren, die das Motivrepertoire variieren und auf der Suche nach der endgültigen Komposition sind. ${ }^{32}$ Doch die Endfassung überbietet die drei Versuche bei weitem. Vor allem findet sich ein ganz neues Motiv für Aktäon, im Grunde genommen aber auch für Diana. Dabei scheint Gainsborough sich entschieden 


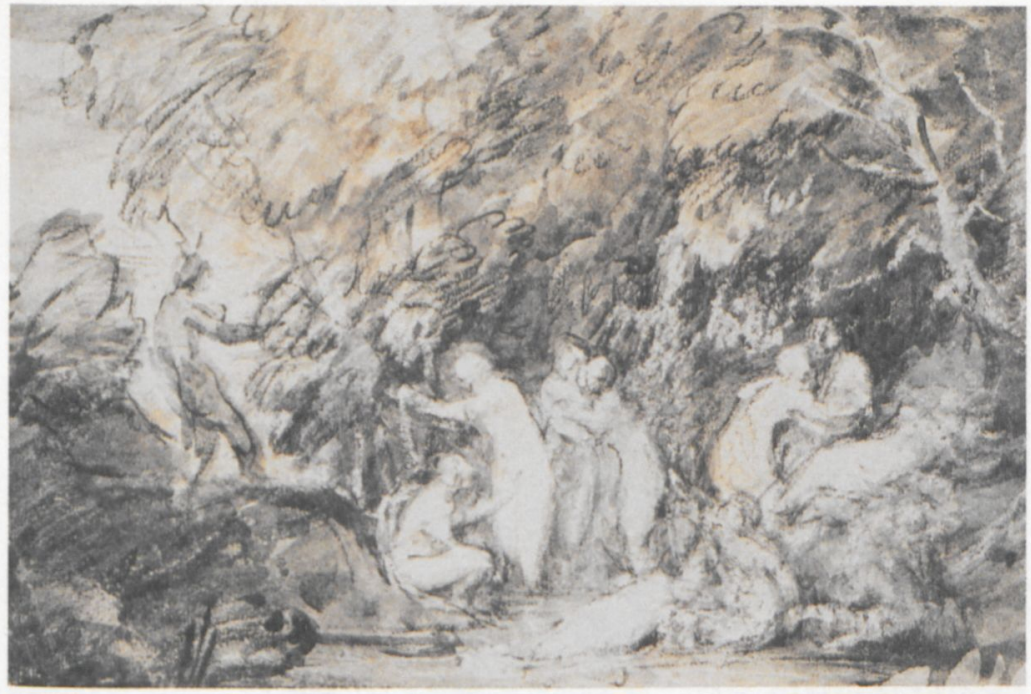

kundig gemacht zu haben, er beherrscht die traditionelle Ikonographie des Themas bis ins Detail. Die Naturgrotte ist da, die aufgescheuchten, sich vergeblich zu bedecken suchenden Nymphen, Diana mit der Mondsichel spritzt das verwandelnde Wasser, Aktäon sprießt bereits das Geweih. Gewänder und Hunde sind über das Bild verteilt. Selbst bestimmte Figurenmotive der klassischen Tradition, wie die linke vordere, sich umschauende Nymphe mit dem übergeschlagenen Bein, die ein Grundmotiv des antiken Dornausziehers variiert, sind ihm geläufig. Sogar die Markierung der Göttin durch einen großen Baum, ein Motiv, das letztlich von Tizian stammt, findet sich bei Gainsborough. "No mistake", hätte der Akademiker feststellen können.

Irritierend bleibt allein die Malweise und bleibt Aktäons Pose. Statt zurückzuschrecken, wie in einer der Gouachen [Abb. 8], beugt er sich vor, kreuzt die Arme, scheint seinen Blick in den Dianens zu versenken, und sie, statt zornblitzend das Wasser zu spritzen, scheint milde Wasser zu spenden. Diese figürliche Konstellation, besonders durch die gekreuzten Arme des Aktäon, stellt das Schema der Taufe Christi dar; man braucht es nur mit Murillo [Abb. 9] zu vergleichen, von dem Gainsborough ein Bild besaß und den er besonders verehrte. ${ }^{33}$ Was also ist bei Gainsborough dargestellt? Auch eine Form der Erkenntnis, nämlich die auf Erden nicht mögliche Liebe zwischen der auf Keuschheit verpflichteten Göttin und dem mythischen Jäger in schicksalhafter Begegnung. Das Wasser der Verwandlung, die Taufe-das ist durchaus tiefsinnig

8 Thomas Gainsborough, Diana und Aktäon, 1784-86, 2. Gouache, $27,9 \times 36,8 \mathrm{~cm}$, Los Angeles, The Huntington Library, Art Collections and Botanical Gardens

C 


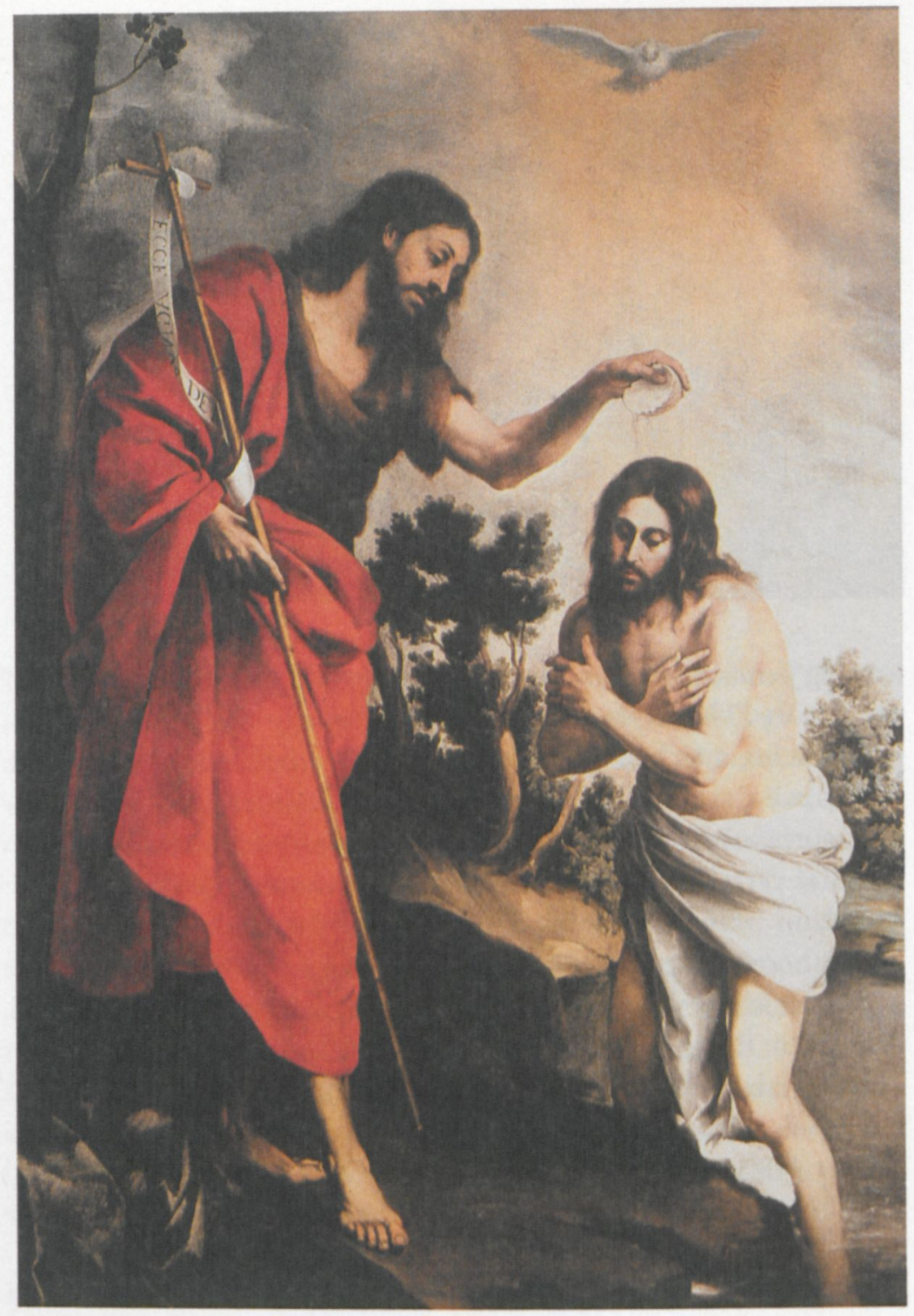

9 Bartolomé Esteban Murillo, Die Taufe Christi, 1655 , Öl a.L., $233 \times 160 \mathrm{~cm}$, Staatliche Museen zu Berlin, Gemäldegalerie gedacht-ist die einzige Möglichkeit der Annäherung von Göttin und Menschenkind, ja, wir werden im Bilde ihrer Verschmelzung, ihrer Vereinigung gewahr. Die Malerei führt uns Aktäons Übergehen in Natur vor, die Grisaillefassung des Bildes verstärkt den Eindruck, Natur und Aktäon sind von einer Farbe und Materie. Malerei wird nicht nur zur Metapher der Metamorphose; vielmehr taucht Aktäon anschaulich in die Natur ein, indem er sich in künstlerische Textur auflöst. In der Tat wird uns der Weg vom 
Text zur Textur demonstriert. Und Diana in ihrer Rolle als vielbrüstige Diana von Ephesus verkörpert bekanntlich nichts anderes als Natura. ${ }^{34}$ Das vermeintlich Unvollendete, besonders auf der rechten Seite, zeigt den Übergang, den wir in beide Richtungen lesen können, besonders deutlich: vom Gegenständlichen in Malerei und von der Malerei zu Gegenständlichem. Noch stärker als bei Tizians spätem Bild, das Gainsborough wohl gekannt hat-es war, wie die beiden anderen Dianenbilder, schon im 18. Jahrhundert 


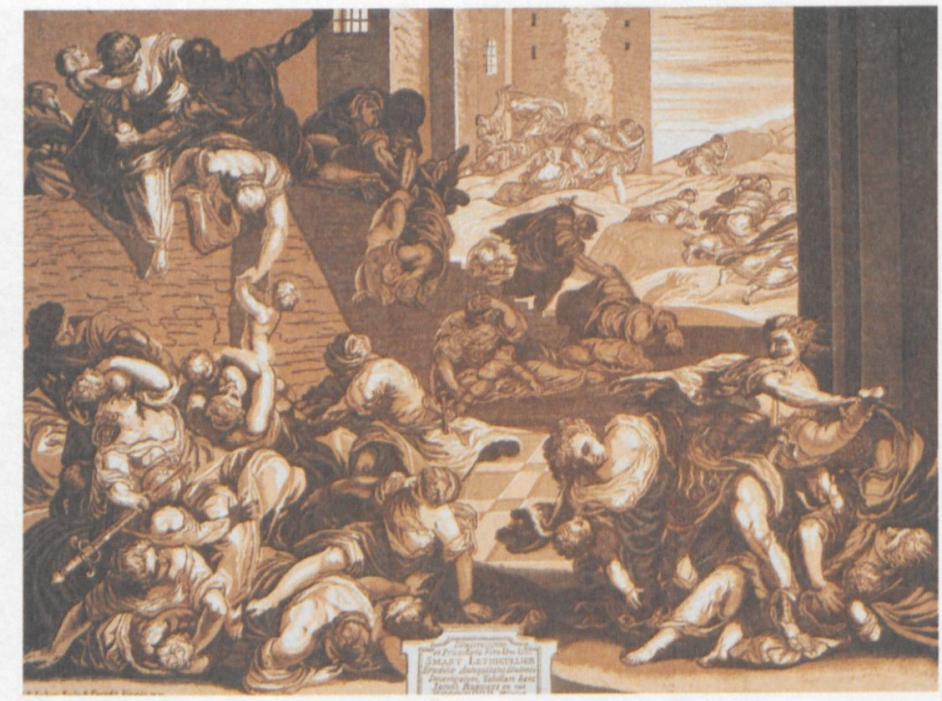

10 John Baptist Jackson, Kindermord zu Bethlehem nach Tintoretto, 1739, Clairobscur-Holzschnitt, $39,4 \times 53,3 \mathrm{~cm}$, Berlin, Privatbesitz in England-, müssen wir projizieren, um Gegenständliches zu schaffen bzw. die Metamorphose zu vollziehen. Insofern werden wir uns unserer Projektionsleistung bewusst und auf die Reflexion über das Wesen der Malerei gestoßen. Hinzu kommt, dass der mittlere Ton des Bildes, die Ockerfarbe, aus Erde, aus dem Schoß der Natur, hergestellt wird.

Die Geschichte des Chiaroscuro, des Helldunkels, als Grundlage eines alternativen Kunstmodells lässt sich vom Clairobscurholzschnitt des 16. Jahrhunderts-das erste italienische Beispiel stellt bezeichnenderweise eine Reproduktion nach Tizian dar ${ }^{35}$ - bis hin zur Etablierung der verschiedensten graphischen Techniken im 18. Jahrhundert verfolgen. Zugleich folgt man dabei der Geschichte des Sehens. Aber nicht darum soll es hier gehen, sondern um die an sich paradoxe Wiederbelebung des Clairobscurholzschnitts im 18. Jahrhundert; rein technisch gesehen war der Clairobscurholzschnitt durch die neuen graphischen Techniken wie Mezzotinto, Aquatinta, Stippel oder auch »vernis mou«, also Weichgrundätzung, längst überholt, was die Differenzierungsmöglichkeiten in der Wiedergabe angeht. Der zweifellos kreativste Clairobscurholzschneider des 18. Jahrhunderts ist John Baptist Jackson. Da er auch über das Medium reflektiert hat, sei allein auf ihn abgehoben. ${ }^{36}$ Nach seiner ersten englischen Zeit ging Jackson nach $\mathrm{Pa}-$ ris, suchte dort mit seiner neuen Technik Fuß zu fassen, der Comte de Caylus versuchte, ihn für das berühmte »Recueil Crozat « zu gewinnen, Jackson lieferte Proben, doch Crozat selbst scheint 


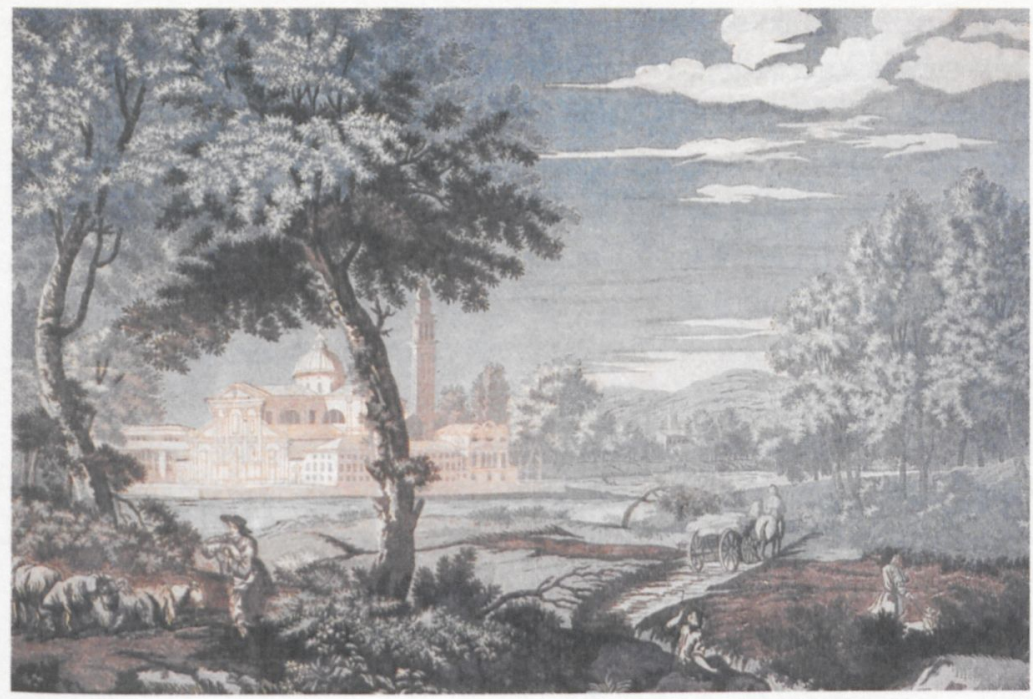

die Mitarbeit verhindert zu haben. Der sehr schwierige Jackson scheint sich nicht nur Freunde gemacht zu haben. Er ging Anfang der 1730er Jahre nach Venedig, hatte sofort Kontakt zu Zanetti, der ab den zwanziger Jahren in Clairobscur schnitt, und zwar primär, wie schon Ugo da Carpi im 16. Jahrhundert, nach den Zeichnungen Parmigianinos, auch dies scheint nicht sehr lange gut gegangen zu sein.

In Venedig jedoch erhielt Jackson aus dem Zanetti-Kreis heraus einen wichtigen Auftrag. Treibende Kraft war hier der englische Gesandte Konsul Smith, dessen Palast am Canale Grande das Zentrum der venezianischen Aufklärung darstellte. Ab 1739 schnitt Jackson 24 Blöcke für 17 Bilder, und zwar nach Gemälden der wichtigsten venezianischen Künstler des 16. Jahrhunderts [Abb. 10]. Die Serie war 1743 fertig und wurde 1745 in gebundener Form publiziert. ${ }^{37} 1744$ schnitt Jackson nach sechs Gouachegemälden von Marco Ricci [Abb. 11] und druckte sie mit sieben bis zehn Blöcken in je individueller Farbe, so dass hier die ersten Faksimileversuche nach Gemälden vorliegen. ${ }^{38}$

Was macht das Besondere all dieser Reproduktionen aus, die zu drucken außerordentlich schwer waren? Jackson ließ sich eigene Pressen bauen nach dem Rollverfahren, entwarf 1730 eine eigene Zylinderpresse, die in der Lage war, mit stärkerem Druck zu arbeiten als alle Graphikpressen zuvor. Traditionellerweise schnitt man zuerst einen Umrissblock, dann die eigentlichen Tonplatten, druckte dann in einem Rahmen erst die Tonplatten, zum Schluss

11 John Baptist Jackson, Landschaft mit $S$. Giorgio Maggiore nach Marco Ricci, 1744, Clair-obscur-Holzschnitt, $41,8 \times 58,5 \mathrm{~cm}$, Braunschweig, Herzog Anton Ulrich-Museum, Kupferstichkabinett 
den Umrissblock, so dass die Linien dadurch, dass sie über die Farben gedruckt wurden, hervortraten. Jackson verzichtete soweit als möglich auf den Umrissblock, besonders bei den Reproduktionen nach den Gouachen von Ricci. Er druckte, um überzeugende Flächentöne zu erhalten, mit feuchtem, in den Rahmen gespanntem Papier. Die Blöcke mussten schnell nacheinander gedruckt werden, wobei es auch möglich war, Farbblöcke teilweise übereinander zu drucken, um auf diese Weise weitere Töne als Mischung zu erhalten. Das Drucken war voller Risiko, viele Abzüge misslangen.

Doch Jackson tat noch etwas anderes. Er druckte mit seiner starken Presse bei einigen Blättern der Gemäldeserie und bei allen Ricci-Nachbildungen zum Schluss mit ein bis zwei Blindstöcken, also ohne Farbe, in das erneut befeuchtete Papier, um ihm ein besonderes, durchaus erhabenes Relief oder Profil einzuprägen. Die Wirkung ist absolut verblüffend, denn diese farblosen Blöcke setzen dem Blatt Lichter auf, und da, wo das Relief in das Blatt einsinkt, bilden sich zusätzliche Schatten, auch gerade in den zuvor schon farbig gedruckten Flächen. Das ähnelt entschieden Tizians Malverfahren mit der groben Leinwand und den trockenen Pigmenten, die nur auf den erhöhten Strukturen der Leinwand aufsitzen. ${ }^{39}$ In beiden Fällen führen die Verfahren zu einer extremen Verlebendigung der Fläche, die zu wogen und zu atmen scheint. Man kann das Jacksonsche Verfahren auch als eine Vorstufe des fotografischen Rasters begreifen. Das rilievo, das die Engländer »embossing « nennen, kann auch rein ornamentale Funktion übernehmen; kein Wunder, dass Jackson später Papiertapeten nach diesem Verfahren gedruckt hat.

Doch ist mit diesen Hinweisen auf die technischen Neuerungen die Bedeutung von Jacksons Blättern noch keineswegs vollständig benannt. Er hat zu seiner besonderen Kunst ein kleines Traktat geschrieben, das 1754 unter dem Titel An Essay on the Invention of Engraving and Printing in Chiaro Oscuro mit acht Bildbeispielen erschien. Nicht nur, dass er die gesamte Geschichte des Clairobscurholzschnitts genau kannte und referiert, er wusste auch, worin der besondere Wert seiner Holzschnitte lag. Man kann es mit einem englischen Begriff sagen, er liegt in der »boldness", seiner besonderen Kühnheit, ja, Dreistigkeit. Denn mitnichten sucht er kleinteilig die Details der Vorlage wiederzugeben. In seinem Essay heißt es: Die »delicate Finishings, and minute Strokes« des Kupferstichs fänden sich im Clairobscurholzschnitt nicht, dafür eine meisterliche und freie Zeichnung: »[...] a boldness of Engraving 
and Relief, which pleases a true taste more than all the little Exactness found in Engravings on Copper Plates«. Feine penible Striche seien etwas für den kleinen Geschmack. »Freedom, Life and Spirit« vermag für die einzelne Figur und die gesamte Komposition nur eine großzügige, sich frei dem Arbeitsprozess überlassende Behandlung zu erzielen. ${ }^{40}$

Was kann aus diesen Überlegungen geschlossen werden? Der Clairobscurholzschnitt sucht in der Tonalität nicht nur ein Äquivalent für Farbigkeit, sondern in erster Linie haucht er der Darstellung Leben ein, durch das "embossing «, aber mehr noch durch die »boldness". Offenbar ist dem Künstler im Zeitalter der Wahrnehmungs- und Wirkungsästhetik klar, dass Ähnlichkeit auch durch Unähnlichkeit zu erzielen ist, dass jede Reproduktion eine Übersetzung darstellt, nicht nur in ein anderes Medium, sondern auch in einen anderen Wahrnehmungszusammenhang, und dass man diesen gezielt avisieren kann, indem man die Möglichkeiten des jeweiligen Mediums auslotet. ${ }^{41}$ Das Äquivalent kann wahrer sein als die Kopie. Aus diesen Erfahrungen konnten die Mezzotintound Aquatintakünstler bis zu Constable und Turner aufbauen.

Eine abschließende Bemerkung. Was hier ansatzweise vorgeführt wurde, war die geraffte Geschichte der Etablierung einer Kunstauffassung, die in der ostentativen Vorführung ihrer medialen Möglichkeiten der Tradition des ut pictura poesis ein Gegenmodell entgegengesetzt hat. Sie erzählt nicht in Analogie zu einer textlichen, logischen Abfolge, sondern führt Strukturen vor Augen, die in ihrer Gesamtheit einen Eindruck erwecken, einen Ton anschlagen, der in den Aufnehmenden weiter klingt und aus dem sie erst die eine oder andere Melodie machen können, angeregt durch die Vorgabe. Kunst wird hier als Erscheinung und als Form der Verlebendigung begriffen und erweist darin ihren Wert. 


\section{Endnoten}

1 Zum klassischen Bildbegriff: Puttfarken, Thomas, Roger de Piles' Theory of Art, New Haven/London, 1985, dort S. 10 zu Poussin mit Lit.; Körner, Hans, Auf der Suche nach der mwahren " Einheit. Ganzheitsvorstellungen in der französischen Malerei und Kunstliteratur vom mittleren 17. bis zum mittleren 19. Jahrhundert, München, 1988; Puttfarken, Thomas, The Discovery of Pictorial Composition. Theories of visual order in painting 1400-1800, New Haven/London, 2000.

2 Zum Verhältnis klassisch-unklassisch als einem rhetorischen Modell: Busch, Werner, "Klassizismus, Klassik", in: Historisches Wörterbuch der Rhetorik, hg.v. Gert Ueding, Bd.4, Tübingen 1998, Sp. 1070-1081; zur Disegno-Theorie: Kemp, Wolfgang, "Disegno. Beiträge zur Geschichte des Begriffs zwischen 1547 und 1607 «, in: Marburger Jahrbuch für Kunstwissenschaft, 19, 1974, S. 219-240.

3 Zu Marsyas: Neumann, Jaromir, Tizian. Die Schindung des Marsyas, Prag, 1962; Bohde, Daniela, Haut, Fleisch und Farbe. Körperlichkeit und Materialität in den Gemälden Tizians, Emsdetten/Berlin, 2001, S.297-342; Kat.Ausst. Der späte Tizian und die Sinnlichkeit der Malerei, hg.v. Sylvia Ferino-Pagden, Kunsthistorisches Museum Wien, Gallerie dell'Accademia Venedig/Wien, 2007, Kat. Nr. 2.13, S.272-275. Zur Pietà: Bohde, Daniela, Haut, Fleisch und Farbe, S.68-89; Kat. Ausst. Der späte Tizian und die Sinnlichkeit der Malerei, Kat. Nr.3.22, S.354-357.

4 Neben den in Anm. 3 zitierten Arbeiten zu Farbe, Werkprozess und dem »Unvollendeten bei Tizian vor allem: von Rosen, Valeska, Mimesis und Selbstbezüglichkeit in Werken Tizians, Emsdetten/Berlin, 2001.

$5 \mathrm{Zu}$ den Bildern der »Poesia«: Rosand, David, »Ut Pictor Poeta. Meaning in Titian's Poesie«, in: New Literary History, 3, 1971/72, S. 527-46; Curto Tanner, Marie, The "Poesie» for Philipp II., Ph. D., New York, 1976; Clinton Nash, Jane, Titian's »Poesie« for Philipp II., Ph. D., The Johns Hopkins University, 1981; Rearick, William Roger, »Titian's Later Mythologies«, in: artibus et historiae 33, 1996, S.23-67 (mit älterer Lit., dort S.63 f. zu den im folgenden erwähnten Änderungen des Aktäon-Bildes während des Werkprozesses); Kat. Ausst. Der späte Tizian..., (s.Anm. 3), S.219-222.

6 Ausführlich zum Thema und zur Exegese: Busch, Werner, "Das keusche und das unkeusche Sehen. Rembrandts Aktaion und Callisto «, in: Zeitschrift für Kunstgeschichte, 52, 1989, S. 257 - 277.Zu den Dianenbildern von Tizian zuletzt: Lawson, James, nTitian's Diana pictures, the passing of an epoch", in: artibus et historiae, 25, 49, 2004, S. 49-63.

$7 \mathrm{Zu}$ den Ovid-Illustrationen: Panofsky, Erwin, Problems in Titian. Mostly iconograhic, London, 1969, S. 159-161; Cieri Via, Claudia, "Diana e Atteone. Continuità e variazione di un mito nell'interpretazione di Tiziano «, in: Die Rezeption der Metamorphosen des Ovid in der Neuzeit. Der antike Mythos in Text und Bild, hg.v. Walter, Hermann, Hans-Jürgen Horn, Berlin 1995, S. 150-59 u. Taf.38-41; Kat. Ausst. Der verblümte Sinn. Illustrationen zu den "Metamorphosen « des Ovid, Galerie der Stadt Kornwestheim, 1997, Kat. Nr.36, 62; Thimann, Michael, Lügenhafte Bilder. Ovids "favole « und das Historienbild in der italienischen Renaissance, Göttingen, 2002, bes. S. 119-129, Abb. 21, 24, 25; Dolce, Lodovico, Le trasformationi, Venedig, 1553 und verbesserte 2.Auflage im selben Jahr.

8 Zitiert nach: Alberti, Leon Battista, Della Pittura-Ưber die Malkunst, hg.v. Oskar Bätschmann, Sandra Gianfreda, ${ }^{2}$ Darmstadt, 2007, S. 146/147.

9 Busch (s.Anm.6), S. 269-271; Kliemann, Julian, „Kunst als Bogenschießen: Domenichinos Jagd der Diana in der Galleria Borghese", in: Römisches Jahrbuch der Biblioteca Hertziana, 31, 1996, S.273-312; Herrmann Fiore, Kristina, "La Caccia di Diana: della genesi del depinto, della questione dell'antico e del colore in rapporto della teoria di Padre Matteo Zaccolini«, in: Domenichino, hg.v. Claudio M. Strinati, Mailand, 1996, S. 240-252; Kliemann, Julian, 1 bersaglio dell'arte: la caccia di Domenichino nella Galleria Borghese, Rom, 2001.

10 Ovid, Metamorphosen, übers. u. hg.v. Hermann Breitenbach, Stuttgart, 1988, 3. Buch, Z.157-162. Dolce übersetzt die entscheidende Passage: »Non è la grotta fabbricata 
ad arte, ma ben l'arte imitato ha la natura in: Dolce (s. Anm. 7), zitiert bei Cieri Via (s.Anm.7), S. 151.

11 Ackerman, James S., "The Tuscan/Rustic Order: A study in the Metaphorical Language of Architecture «, in: Journal of the Society of Architectural Historians 42, 1983, S. 15 - 34; Serlio, Sebastiano, "Regole generali (Venedig 1537) ", in: Sebastiano Serlio, Tutte le opere d'architettura, Bd.4, Venedig, 1584, fol. 126; Kris, Ernst, "Der Stil >rustique ‘. Die Verwendung des Naturabgusses bei Wenzel Jamnitzer und Bernard Palissy«, in: Jahrbuch der Kunsthistorischen Sammlungen in Wien, N. F. 1, 1926, S.137-208.

12 Siehe: Busch, Werner, „Aretinos Evokation von Tizians Kunst «, in: Zeitschrift für Kunstgeschichte 63, 1999, bes. S. $98-100$.

13 Ebd., S. 91 f., 98.

14 Dolce, Lodovico, La Poetica d'Horazio, übers. v. Lodovico Dolce, o. O., 1536.

15 Horaz, Ars Poetica-Die Dichtkunst, übers. und mit einem Nachwort von Eckart Schäfer, Stuttgart, 1994, S. 5.

16 Wethey, Harold E., The Paintings of Titian, Bd.3, The Mythological and Historical Paintings, London, 1975, Nr. 8, S.136 ff.; Cocke, Richard, "Titian the Second Apelles. The Death of Actaeon «, in: Renaissance Studies 13, 1999, S.303-311.

17 Von Rosen (s.Anm. 4), bes. Kap.7.

18 Wedgwood Kennedy, Ruth, "Apelles Redivivus «, in: Essays in Memory of Karl Lehmann, hg.v. Lucy Freemann Sandler, New York, 1964, S. 160-170.

19 Caius Plinius Secundus d.Ä., Naturkunde-Historia Naturalis, Buch 35, Farben, Malerei, Plastik, hrsg. und übers. von Roderich König in Zusammenarbeit mit Gerhard Winkler, München, 1978, \$50, S. 45 f.; Moffit, John F., »Leonardo's >sfumato und Apelles's satramentum «, in: Paragone, 473, 1989, S. 88-92; Irle, Klaus, "Apelles, Zeuxis, Lysippos und die Malerei des Cinquecento", in: Antiquarische Gelehrsamkeit und Bildende Kunst. Die Gegenwart der Antike in der Renaissance (=Atlas. Bonner Beiträge zur Renaissanceforschung, hg.v. Gunter Schweikhart, Bd. 1), Köln, 1996, S. 123-135.

20 Plinius (s. Anm. 19), $\$ 80$, S. 64 f.

21 Von Rosen (s.Anm. 4), s. Kap. 6 »Eloquente ,Colpi «, S. 299-365.

22 Ovid (s.Anm. 10), 3. Buch, Z. 188-190: „dringend wünschte sie jetzt, zur Hand die Pfeile zu haben / Aber sie nahm, was sie hatte: sie schöpfte vom Wasser und goss/Über des Mannes Gesicht [...]«.

23 Etwa in der venezianischen 1. Volgare-Ausgabe von 1513 (zuerst 1497), fol. 19' , Abb. bei Cieri Via (s.Anm.7), Taf.40a.

24 Ovid (s.Anm. 10), 3. Buch, Z.199; Darstellung der Szene mit den endgültig über Aktäon herfallenden Hunden: Illustration von Bernard Salomon aus: La Metamorphose d'Ovide figurée, Lyon, 1557, Nr.43, Abb. bei Panofsky (s. Anm. 7), Abb. 174.

25 Von Flemming, Victoria, Arma Amoris. Sprachbild und Bildsprache der Liebe. Kardinal Scipione Borghese und die Gemäldezyklen Francesco Albanis (=Berliner Schriften zur Kunst, Bd.6), Mainz, 1996, Abb. 1, S. 21 f.; zur Deutung: Verhayen, Egon, The Paintings in the "Studiolo of Isabella d'Este at Mantua, New York, 1971, S.41-44.

26 Zur Exegese: Busch (s. Anm. 6).

27 Ovid (s.Anm. 10), 3. Buch, Z. 206.

28 Jackson of Exeter, William, The Four Ages together with Essays on Various Subjects, London, 1798, S. 155.

29 Hayes, John (Hg.), The Letters of Thomas Gainsborough, New Haven/London, 2001, S. 40 (Brief 22, an William Jackson, 23. August 1767).

30 "I'am sick of portraits and wish very much to take my Viol da Gam and walk off to some sweet Village where I can paint Landskips [... ] «, ebd., S. 68 (Brief 40, an William Jackson, 4. Juni o. J. [um 1768/69]).

31 Hayes, John, The Drawings of Thomas Gainsborough, 2 Bde., London, 1970.

32 Zum Bild, den Gouachen und der Interpretation: Busch, Werner, Das sentimentalische Bild. Die Krise der Kunst im 18. Jahrhundert und die Geburt der Moderne, München, 1993, 


\section{Endnoten/Abbildungsnachweis}

S.171-180; Kat. Ausst. Gainsborough, hg.v. Michael Rosenthal, Martin Myrone, Tate Britain, London, 2002, Kat. Nr. 173-177, S. 278-281.

33 Abb. bei Busch (s.Anm. 32), Abb.61, S. 175.

34 Kemp, Wolfgang, Natura. Ikonographische Studien zur Geschichte und Verbreitung einer Allegorie, phil. Diss. Tübingen, 1973, Kap.II. 3 „Die Diana Ephesia als Allegorie der Natur«, S. 25-29.

35 ferstichkabinett Staatliche Museen Preussischer Kulturbesitz, Berlin, o. J. (um 1970), Kat. Nr. 5. Der Hl. Hieronymus vor dem Kruzifix, Clairobscurholzschnitt in zwei Platten von Ugo da Carpi.

36 Kainen, Jacob, John Baptist Jackson, 18th Century Master of the Color Woodcut, Washington, 1962; Wöldicke, Evelyn, Die Holzschnitte des John Baptist Jackson. Druckgraphische Werke im Spannungsfeld zwischen Tradition und Innovation, M.A.-Arbeit Freie Universität Berlin, 2004 (Frau Wöldicke arbeitet z.Zt. an einer Dissertation zur Wiederbelebung des Clairobscurholzschnitts im 18. Jahrhundert); Jacksons Traktate: Jackson, John Baptist, An essay on the invention of engraving and printing in chiaro oscuro, as practised by Albert Durer, Hugo di Carpi, \&c. and the application of it to the making paper hangings of taste, duration, and elegance, London, 1754; Jackson, John Baptist, A Lover of Art: An Enquiry into the Origins of Printing in Europe, London, 1752.

37 Titiani Vecelli, Pauli Caliarii, Jacobi Robusti et Jacobi de Ponte; opera selectiora a Joanne Baptista Jackson, Anglo, ligno coelata et coloribus adumbrata, Venedig, 1745.

38 Kainen (s.Anm. 36), Kat. Nr. 38-43; Farbabb. eines Beispieles nach Ricci: Kat. Ausst. Von Cranach bis Baselitz, Meisterwerke des Clairobscur-Holzschnitts, hg.v. Claus Kemmer, Herzog Anton Ulrich-Museum Braunschweig, Kunstmuseum des Landes Niedersachsen, Braunschweig, 2003, Taf. XIV, Kat.Ausst. Nr. 130.

39 Zuletzt zur Technik: Oberthaler, Elke, "Tizians Spätstil an Hand von Nymphe und Schäfer«, in: Der späte Tizian und die Sinnlichkeit der Malerei, Kat. Ausst. (s.Anm. 3), S. $111-121$.

40 Jackson, An Essay (s.Anm.36), S.6.

41 Hierzu: Bann, Stephen, Parallel Lines. Printmakers, Painters and Photographers in Nineteenth-Century France, New Haven/London, 2001; ders., "Der Reproduktionsstich als Ubersetzung«, in: Vorträge aus dem Warburg-Haus, Bd.6, Berlin, 2002, S.41-76

\section{Abbildungsnachweis}

1 Tizian, Die Schindung des Marsyas, um 1570, Ol a. L., $220 \times 204 \mathrm{~cm}$, Erzbistum Olomouc, Erzbischöflicher Palast Kroměřiž, Gemäldegalerie.

2 Tizian, Diana und Aktäon, 1556-59, Ol a.L., 190,3 × 207 cm, Edinburgh, National Gallery of Scotland.

3 Tizian, Diana und Callisto, 1556-59, Ol a. L., $187 \times 205 \mathrm{~cm}$, Edinburgh, National Gallery of Scotland.

4 Tizian, Der Tod des Aktäon, um 1559-1568, Ol a. L., $179 \times 189 \mathrm{~cm}$, London, National Gallery.

5 Ill. zu Ovid, Metamorphosen, Venedig 1513, fol. XIX' ${ }^{\text {, }}$, Holzschnitt, Diana und Aktäon.

6 Ill. zu Ovid, Metamorphosen, Lyon 1557, No.43, Holzschnitt von Bernard Salomon, Der Tod des Aktäon.

7 Thomas Gainsborough, Diana und Aktäon, 1784-86, Ol a.L., 158,1 $\times 188 \mathrm{~cm}$, London, Her Majesty Queen Elizabeth II.

8 Thomas Gainsborough, Diana und Aktäon, 1784-86, 2. Gouache, $27,9 \times 36,8 \mathrm{~cm}$, Los Angeles, The Huntington Library, Art Collections and Botanical Gardens.

9 Bartolomé Esteban Murillo, Die Taufe Christi, 1655, Ol a. L., $233 \times 160 \mathrm{~cm}$, Staatliche Museen zu Berlin, Gemäldegalerie. 
10 John Baptist Jackson, Kindermord zu Bethlehem nach Tintoretto, 1739, Clairobscurholzschnitt, $39,4 \times 53,3 \mathrm{~cm}$, Berlin, Privatbesitz.

11 John Baptist Jackson, Landschaft mit S. Giorgio Maggiore nach Marco Ricci, 1744, Clairobscurholzschnitt, $41,8 \times 58,5 \mathrm{~cm}$, Braunschweig, Herzog Anton Ulrich-Museum, Kupferstichkabinett. 